\title{
ISOPARAMETRIC HYPERSURFACES WITH FOUR PRINCIPAL CURVATURES, II
}

\author{
QUO-SHIN CHI
}

\begin{abstract}
In this sequel to an earlier article, employing more commutative algebra than previously, we show that an isoparametric hypersurface with four principal curvatures and multiplicities $(3,4)$ in $S^{15}$ is one constructed by Ozeki and Takeuchi and Ferus, Karcher, and Münzner, referred to collectively as of OT-FKM type. In fact, this new approach also gives a considerably simpler proof, both structurally and technically, that an isoparametric hypersurface with four principal curvatures in spheres with the multiplicity constraint $m_{2} \geq 2 m_{1}-1$ is of OT-FKM type, which left unsettled exactly the four anomalous multiplicity pairs $(4,5),(3,4),(7,8)$, and $(6,9)$, where the last three are closely tied, respectively, with the quaternion algebra, the octonion algebra, and the complexified octonion algebra, whereas the first stands alone in that it cannot be of OT-FKM type. A by-product of this new approach is that we see that Condition B, introduced by Ozeki and Takeuchi in their construction of inhomogeneous isoparametric hypersurfaces, naturally arises. The cases for the multiplicity pairs $(4,5),(6,9)$, and $(7,8)$ remain open now.
\end{abstract}

\section{$\S 1$. Introduction}

An isoparametric hypersurface in a space form is a complete hypersurface whose principal curvatures and their multiplicities are fixed constants. The long history of the study of isoparametric hypersurfaces dates back to 1918, when isoparametric surfaces in Euclidean 3-space arose in the study of geometric optics (see [14], [22], [20]); in contrast, their latest application to integrable systems came as late as 1995 (see [11]), to the author's knowledge. The classification problem of isoparametric hypersurfaces started when Segre [21], for ambient dimension 3, and Levi-Civita [15], for arbitrary ambient dimension $n$, classified such hypersurfaces in Euclidean space; they are none other than the cylinders $S^{k} \times \mathbb{R}^{n-k-1}$. Cartan [2] then

Received May 2010. Accepted May 12, 2011.

2010 Mathematics Subject Classification. Primary 53C40.

The author was partially supported by National Science Foundation grant DMS0103838 .

(C) 2011 by The Editorial Board of the Nagoya Mathematical Journal 
took up the task and quickly settled the hyperbolic case; again, the hyperbolic cylinders $S^{k} \times H^{n-k-1}$ are the only ones. The spherical case amazed Cartan, as such hypersurfaces displayed remarkably deep properties. In fact, he classified the case when the number $g$ of principal curvatures is at most 3. Clifford tori $S^{k}(r) \times S^{n-k-1}(s) \subset S^{n}, r^{2}+s^{2}=1$, constitute the case when $g=2$. For $g=3$, he showed that such hypersurfaces are tubes of constant radii around the Veronese embedding of the projective plane $\mathbb{F} P^{2}$ in $S^{3 m+1}$, where $m=1,2,4$, or 8 is the dimension of the standard normed algebra $\mathbb{F}=\mathbb{R}, \mathbb{C}, \mathbb{H}$, or the Cayley algebra $\mathbb{O}$, respectively (see [3], [4]). In particular, this gives a very geometric description of the Cayley plane that appears in the classification of the rank 1 symmetric spaces of compact type that Cartan had classified earlier using Lie group theory he developed. Therefore, all these isoparametric hypersurfaces are homogeneous.

To make a long story short, leaving the beautiful subsequent development of the classification problem to the introductory section in [6], let us simply remark that by [18] it holds that $g$ can only be $1,2,3,4,6$; moreover, the principal values have at most two multiplicities. In fact, if we list these principal values as $\lambda_{1}<\cdots<\lambda_{g}$, and their associated multiplicities as $m_{1}, \ldots, m_{g}$, respectively, then $m_{i}=m_{i+2}$, where the subscripts are modulo $g$. In particular, $m_{1}=m_{2}$ (actually, $=1,2,4$, or 8 associated with the standard normed algebras) when $g=3$. We will denote these two multiplicities by $m_{1}$ and $m_{2}$, with the understanding that $m_{1} \leq m_{2}$. To each isoparametric hypersurface in the sphere, there is indeed a 1-parameter family of isoparametric hypersurfaces in the sphere that degenerates to two submanifolds of the sphere of codimensions $m_{1}+1$ and $m_{2}+1$, called the focal manifolds of the isoparametric hypersurface and denoted by $M_{+}$and $M_{-}$, respectively. The principal values of the focal manifold of codimension $m_{1}+1$ are $0,1,-1$ with multiplicities $m_{1}, m_{2}, m_{2}$, respectively, with $m_{1}$ and $m_{2}$ interchanged for the other focal manifold.

It is known (see [1]) that $m_{1}=m_{2}=1$ or 2 in the case $g=6$. The case $m_{1}=m_{2}=1$ was settled by Dorfmeister and Neher [10] (see also Miyaoka [16]). The other case has recently been settled by Miyaoka [17]. Such isoparametric hypersurfaces are again homogeneous.

Cartan [5] found two homogeneous examples of isoparametric hypersurfaces with $g=4$ and $\left(m_{1}, m_{2}\right)=(1,1)$ or $(2,2)$. He indicated without proof that his example with multiplicity pair $(2,2)$ is the only isoparametric hypersurface with the given $g$ and multiplicities; an outline of a proof was given by Ozeki and Takeuchi in [19, Part II, pp. 53-54]. 
In contrast, inhomogeneous examples do appear in the case $g=4$. Ozeki and Takeuchi [19, Part I, pp. 541, 549] introduced the notions of Conditions $\mathrm{A}$ and $\mathrm{B}$ to first construct two families of them [19, Part I], followed by Ferus, Karcher, and Münzner's [12] generalization to infinite families using Clifford representations, where $m_{1}$ and $m_{2}$ are explicitly given in terms of the dimensions of irreducible Clifford modules. We refer to these examples collectively as of OT-FKM type. These examples, together with the two homogeneous examples with $\left(m_{1}, m_{2}\right)=(2,2)$ and $(4,5)$, not of OT-FKM type, have constituted all known isoparametric hypersurfaces with four principal curvatures in spheres. In fact, using homotopy theory, Stolz [23] proved that the multiplicity pair of an isoparametric hypersurface with $g=4$ in the sphere is $(2,2),(4,5)$, or one of those of isoparametric hypersurfaces of OTFKM type. In [6], we classified that, except for possibly the four cases with $\left(m_{1}, m_{2}\right)=(4,5),(3,4),(6,9)$, or $(7,8)$, all other isoparametric hypersurfaces with $\left(m_{1}, m_{2}\right) \neq(2,2)$ are necessarily of OT-FKM type.

It is worth pointing out that isoparametric hypersurfaces of OT-FKM type with multiplicity pairs $\left(m_{1}, m_{2}\right)=(3,4),(7,8)$, and $(6,9)$ are anomalous in the sense that they are the only ones for which the two symmetric Clifford algebras $C_{m_{1}+1}^{\prime}$ and $C_{m_{2}+1}^{\prime}$ can act on $\mathbb{R}^{2 m_{1}+2 m_{2}+2}$ to produce incongruent isoparametric hypersurfaces in $S^{2 m_{1}+2 m_{2}+1}$ (see [12]). Their isoparametric structures are tied, respectively, with $\mathbb{H}, \mathbb{O}$, and $\mathbb{O}(\mathbb{C})$ (see [19]). On the other hand, an isoparametric hypersurface with $g=4$ and multiplicities $\left(m_{1}, m_{2}\right)=(4,5)$ stands alone, as it cannot be of OT-FKM type.

The classification theorem in [6] (see also [7]), where it is assumed that $m_{2} \geq 2 m_{1}-1$, eventually comes down to an estimate on the dimension of certain singular varieties associated with the complexified second fundamental form of the focal manifold of codimension $m_{1}+1$, restricted to the direct sum of the two eigenspaces of the principal values $1,-1$. This restriction to a subspace of a typical tangent space of the focal manifold complicates the dimension estimate, since the restriction does not preserve the constant rank that the second fundamental matrix of a focal manifold enjoys. Moreover, the tracking of the interplay between real and complex varieties in the proof brings in additional technicality.

The primary goal in this sequel to [6] is to prove that an isoparametric hypersurface with $g=4$ and $\left(m_{1}, m_{2}\right)=(3,4)$ is one of OT-FKM type as well, which leaves only three multiplicity pairs $(4,5),(6,9)$, and $(7,8)$ unsettled. In fact, this grew out of an attempt to look at the classification theorem in [6] from a different angle. 
Indeed, employing more commutative algebra than that explored in [6], we first present a considerably simpler proof of the classification theorem in [6] by investigating the (complexified) second fundamental form itself without further restriction. An advantage of this approach is that we see that Condition B of Ozeki and Takeuchi, which all isoparametric hypersurfaces of OT-FKM type enjoy as a matter of fact, naturally arises.

The key ingredient on which this new proof of the classification theorem depends is the satisfying result (Proposition 4) that for $m_{1}<m_{2}$, if the components $p_{0}, p_{1}, \ldots, p_{m_{1}}$ of the second fundamental form of $M_{+}$ form a regular sequence in the ring of polynomials in $m_{1}+2 m_{2}$ variables (over the complex numbers), then the isoparametric hypersurface is of OTFKM type. The result sheds light on why the remaining multiplicity pairs $(3,4),(6,9)$, and $(7,8)$ are anomalous. It is because in these cases $p_{0}, \ldots, p_{m_{1}}$ no longer form a regular sequence in general, due to the aforementioned fact that incongruent isoparametric hypersurfaces of OT-FKM type do occur in $S^{2 m_{1}+2 m_{2}+1}$; the zero locus cut out by a nonregular sequence $p_{0}, \ldots, p_{m_{1}}$ can be wildly untamed, even in the complex category. In fact, Proposition 4 follows from one of the 10 identities defining an isoparametric hypersurface (see [19, Part I, p. 530]). Namely,

$$
\sum_{a=0}^{m_{1}} p_{a} q_{a}=0
$$

where $q_{0}, q_{1}, \ldots, q_{m_{1}}$ are the components of the third fundamental form of $M_{+}$. That $p_{0}, \ldots, p_{m_{1}}$ form a regular sequence ensures that

$$
q_{a}=\sum_{b=0}^{m_{1}} r_{a b} p_{b},
$$

where $0 \leq a \leq m_{1}$, for some linear homogeneous polynomials $r_{a b}$ satisfying

$$
r_{a b}=-r_{b a}
$$

for $0 \leq a, b \leq m_{1}$. This is exactly Condition B of Ozeki and Takeuchi, from which [6, Proposition 19, (8.1)-(8.3)] readily follows. The mild condition $m_{1}<m_{2}$ then warrants that $[6,(8.4)]$ also holds true; the isoparametric hypersurface is then of OT-FKM type (see [6], [7]). That $p_{0}, \ldots, p_{m_{1}}$ indeed form a regular sequence, when $m_{2} \geq 2 m_{1}-1$, is a consequence of a rather straightforward dimension estimate, facilitated by the constant rank of the 
shape operators of $M_{+}$, over the subset where the Jacobian matrix of these polynomials fails to be of rank $m_{1}+1$ on the variety cut out by them.

What is more important is that in fact the new approach in this paper provides us with a proof that an isoparametric hypersurface with $g=4$ and $\left(m_{1}, m_{2}\right)=(3,4)$ in $S^{15}$ is of OT-FKM type, by showing that there exist on $M_{+}$points of Condition A of Ozeki and Takeuchi. The conclusion then follows from the result of Dorfmeister and Neher [9] (see also [8]) which states that Condition A alone implies that the isoparametric hypersurface is of OT-FKM type.

The salient feature of this approach would appear applicable also to the case $\left(m_{1}, m_{2}\right)=(7,8)$. We comment on this in the concluding remarks.

\section{$\S 2$. The background commutative algebra}

Recall from [13, p. 152] that a regular sequence in a commutative ring $R$ with identity is a sequence $a_{1}, \ldots, a_{k}$ in $R$ such that the ideal $\left(a_{1}, \ldots, a_{k}\right)$ is not $R$ and, moreover, that $a_{1}$ is not a zero divisor in $R$ and $a_{i+1}$ is not a zero divisor in the quotient ring $R /\left(a_{1}, \ldots, a_{i}\right)$ for $1 \leq i \leq k-1$.

There is a powerful property in commutative algebra that dictates the algebraic independence of a regular sequence (see [13, Proposition 5.10, p. 152]), which is specialized to fit our purpose in the following proposition.

Proposition 1. Let $a_{1}, \ldots, a_{k}$ be a regular sequence in $R$. Then for any homogeneous polynomial $F\left(t_{1}, \ldots, t_{k}\right)$ in $k$ variables over $R$ with $F\left(a_{1}, \ldots\right.$, $\left.a_{k}\right)=0$, there always holds that all the coefficients of $F$ belong to $\left(a_{1}, \ldots, a_{k}\right)$.

For the convenience of the reader, let us recall a crucial inductive procedure in [6, Proposition 39, p. 57] to generate regular sequences in a polynomial ring.

Proposition 2. Over the complex numbers, if $p_{1}, \ldots, p_{k}, k \geq 2$, are linearly independent homogeneous polynomials of equal degree $\geq 1$ in a polynomial ring $P$ such that the ideal $\left(p_{1}, \ldots, p_{k-1}\right)$ is prime and that $p_{1}, \ldots, p_{k-1}$ form a regular sequence in $P$, then $p_{1}, \ldots, p_{k}$ form a regular sequence in $P$.

To warrant the primeness of an ideal $\left(p_{1}, \ldots, p_{s}\right)$ in a polynomial ring, the following (see [6, Proposition 43, p. 59]) is essential.

Proposition 3. Over the complex numbers, let $p_{1}, \ldots, p_{s}$ be a regular sequence of homogeneous polynomials in a polynomial ring, let $V$ be the variety defined by $p_{1}=\cdots=p_{s}=0$, and let $J$ be the subvariety of $V$ where 
the rank of the Jacobian matrix of $p_{1}, \ldots, p_{s}$ is $<s$. If $\operatorname{dim}(J) \leq \operatorname{dim}(V)-2$, then the ideal $\left(p_{1}, \ldots, p_{s}\right)$ is prime.

Note that the homogeneity of $p_{1}, \ldots, p_{s}$ is to guarantee that the variety $V$ is connected.

Corollary 1 . Over the complex numbers, let $p_{1}, \ldots, p_{k}, k \geq 2$, be linearly independent homogeneous polynomials of equal degree $\geq 1$ in a polynomial ring. For $i \leq k$, let $V_{i}$ be the variety defined by $p_{1}=\cdots=p_{i}=0$, and let $J_{i}$ be the subvariety of $V_{i}$ where the rank of the Jacobian matrix of $p_{1}, \ldots, p_{i}$ is $<i$. If $\operatorname{dim}\left(J_{i}\right) \leq \operatorname{dim}\left(V_{i}\right)-2$ for $1 \leq i \leq k-1$, then $p_{1}, \ldots, p_{k}$ form a regular sequence in the polynomial ring.

For a proof, note that $p_{1}$ clearly forms a regular sequence, so that Proposition 3 implies that $\left(p_{1}\right)$ is a prime ideal, so the corollary is true when $i=1$. Repeated applications of Propositions 2 and 3 then deduce that the ideal $\left(p_{1}, \ldots, p_{i}\right)$ is prime and that $p_{1}, \ldots, p_{i}$ form a regular sequence for $1 \leq i \leq k-1$. From this, Proposition 2 gives that $p_{1}, \ldots, p_{k}$ form a regular sequence in the polynomial ring.

\section{$\S 3$. The game plan}

We will follow closely the notation in [6] for ease of exposition. Let $n_{0}, n_{1}, \ldots, n_{m_{1}}$ be an orthonormal basis of a typical normal space to the focal manifold $M_{+}$of codimension $m_{1}+1$. Let $p_{0}, p_{1}, \ldots, p_{m_{1}}$ be the associated symmetric quadratic forms associated with the second fundamental form $S$ of $M_{+}$. That is,

$$
p_{a}(X):=\left\langle S(X, X), n_{a}\right\rangle / 2
$$

for $0 \leq a \leq m_{1}$. Let $q_{0}, q_{1}, \ldots, q_{m_{1}}$ be the associated symmetric cubic forms of the third fundamental form of $M_{+}$. That is,

$$
q_{a}(X):=\left\langle Q(X, X, X), n_{a}\right\rangle / 3
$$

where

$$
\begin{aligned}
Q(X, Y, Z) & :=\left(D_{X}^{\perp} S\right)(Y, Z) \\
& =D_{X}^{\perp}(S(Y, Z))-S\left(\nabla_{X} Y, Z\right)-S\left(Y, \nabla_{X} Z\right)
\end{aligned}
$$

for $0 \leq a \leq m_{1}$, where $D^{\perp}$ is the normal connection and $\nabla$ is the Riemannian connection of $M_{+}$. These homogeneous polynomials belong to the polynomial ring $\mathcal{P}$ in $m_{1}+2 m_{2}$ variables corresponding to the dimension of $M_{+}$. 
To apply Proposition 1 , suppose that we have established that $p_{0}, p_{1}, \ldots$, $p_{m_{1}}$ form a regular sequence in $\mathcal{P}$.

Let us recall an identity of Ozeki and Takeuchi [19, Part I, p. 530]. Namely,

$$
p_{0} q_{0}+p_{1} q_{1}+\cdots+p_{m_{1}} q_{m_{1}}=0 .
$$

We can interpret (2) in the spirit of Proposition 1 above. Namely, consider the homogeneous polynomial

$$
F\left(t_{0}, t_{1}, \ldots, t_{m_{1}}\right)=q_{0} t_{0}+q_{1} t_{1}+\cdots+q_{m_{1}} t_{m_{1}}
$$

over the ring $\mathcal{P}$. Since by assumption $p_{0}, p_{1}, \ldots, p_{m_{1}}$ form a regular sequence with $F\left(p_{0}, p_{1}, \ldots, p_{m_{1}}\right)=0$ by $(2)$, it follows from Proposition 1 that $q_{0}, q_{1}$, $\ldots, q_{m_{1}}$ belong to the ideal $\left(p_{0}, p_{1}, \ldots, p_{m_{1}}\right)$. That is, we have

$$
q_{a}=\sum_{b=0}^{m_{1}} r_{a b} p_{b}
$$

for $0 \leq a \leq m_{1}$, where $r_{a b}$ are homogeneous polynomials of degree 1 . Substituting (3) into (2), we obtain

$$
\sum_{a \leq b}\left(r_{a b}+r_{b a}\right) p_{a} p_{b}=0
$$

Considering the homogeneous polynomial

$$
F\left(t_{0}, t_{1}, \ldots, t_{m_{1}}\right)=\sum_{a \leq b}\left(r_{a b}+r_{b a}\right) t_{a} t_{b}
$$

over the ring $\mathcal{P}$ and observing that $F\left(p_{0}, p_{1}, \ldots, p_{m_{1}}\right)=0$ by (4), Proposition 1 implies that $r_{a b}+r_{b a}$ belongs to the ideal $\left(p_{0}, p_{1}, \ldots, p_{m_{1}}\right)$. However, this forces $r_{a b}+r_{b a}=0$ since it is homogeneous of degree 1 , whereas $p_{0}, p_{1}, \ldots, p_{m_{1}}$ are homogeneous of degree 2 . We thus conclude that in $(3)$ we have

$$
r_{a b}=-r_{b a}
$$

Now we introduce the Euclidean coordinates of the eigenspaces, with eigenvalues $1,-1,0$, of the shape operator $S_{n_{0}}$ to be $u_{\alpha}, 1 \leq \alpha \leq m_{2}, v_{\mu}, 1 \leq$ $\mu \leq m_{2}$, and $w_{p}, 1 \leq p \leq m_{1}$, respectively. Set

$$
r_{a b}:=\sum_{\alpha} T_{a b}^{\alpha} u_{\alpha}+\sum_{\mu} T_{a b}^{\mu} v_{\mu}+\sum_{p} T_{a b}^{p} w_{p} .
$$


By [6, p. 18],

$$
\begin{aligned}
2 p_{0} & =\sum_{\alpha}\left(u_{\alpha}\right)^{2}-\sum_{\mu}\left(v_{\mu}\right)^{2} \\
p_{a} & =\sum_{\alpha \mu} S_{\alpha \mu}^{a} u_{\alpha} v_{\mu}+\sum_{\alpha p} S_{\alpha p}^{a} u_{\alpha} w_{p}+\sum_{\mu p} S_{\mu p}^{a} v_{\mu} w_{p}
\end{aligned}
$$

for $1 \leq a \leq m_{1}$, where we set

$$
S_{\alpha \mu}^{a}:=\left\langle S\left(X_{\alpha}, Y_{\mu}\right), n_{a}\right\rangle
$$

and so forth, with $X_{\alpha}, Y_{\mu}$, and $Z_{p}$ the orthonormal bases for the coordinates $u_{\alpha}, v_{\mu}$, and $w_{p}$, respectively. Note that our $p_{a}$ are different from those in $[6$, (6.6)], which are truncated versions of ours. We claim that

$$
T_{a 0}^{\alpha}=T_{a 0}^{\mu}=0
$$

for $1 \leq a \leq m_{1}$. To this end, we calculate $q_{a}$ in two ways. On the one hand, substituting (6) and (7) into (3), we see that $q_{a}$ has the term

$$
\left(\sum_{\alpha} T_{a 0}^{\alpha} u_{\alpha}\right)\left(\sum_{\beta}\left(u_{\beta}\right)^{2}\right) / 2,
$$

so that the coefficient of $\left(u_{\alpha}\right)^{3}$ in $q_{a}$, denoted by $q_{a}^{\alpha \alpha \alpha}$, is

$$
q_{a}^{\alpha \alpha \alpha}=T_{a 0}^{\alpha} / 2
$$

On the other hand, when calculating $D^{\perp}$, we can pick a normal frame so that the normal connection form is zero at any fixed point. Then by (1) and the fact that $S_{\alpha \beta}^{a}=S^{a}\left(X_{\alpha}, X_{\beta}\right)=0$, we calculate to see that

$$
\begin{aligned}
3 T_{a 0}^{\alpha} / 2 & =3 q_{a}^{\alpha \alpha \alpha}=\left\langle Q\left(X_{\alpha}, X_{\alpha}, X_{\alpha}\right), n_{a}\right\rangle \\
& =d S_{\alpha \alpha}^{a}\left(X_{\alpha}\right)-\sum_{t} \theta_{\alpha}^{t}\left(X_{\alpha}\right) S_{t \alpha}^{a}-\sum_{t} \theta_{\alpha}^{t}\left(X_{\alpha}\right) S_{\alpha t}^{a} \\
& =-2 \sum_{t} \theta_{\alpha}^{t}\left(X_{\alpha}\right) S_{t \alpha}^{a}=-2 \sum_{p} \theta_{\alpha}^{p}\left(X_{\alpha}\right) S_{p \alpha}^{a}-2 \sum_{\mu} \theta_{\alpha}^{\mu}\left(X_{\alpha}\right) S_{\alpha \mu}^{a} \\
& =0
\end{aligned}
$$

where $\theta_{j}^{i}$ is the Riemannian connection forms of $M_{+}$and the last equality is by $[6,(4.18)]$. Likewise, $T_{a 0}^{\mu}=0$. Hence, (8) is proved. The skew-symmetry of $r_{a b}$ in $a, b$ then yields

$$
T_{0 a}^{\alpha}=T_{0 a}^{\mu}=0
$$


Next, let us calculate $q_{0}$ in two ways. On the one hand, we expand $q_{0}$ by (3), (6), (7), and (10), keeping in mind that $q_{0}$ is homogeneous of degree 1 in $u_{\alpha}, v_{\mu}$, and $w_{p}$, by [19, Part I, p. 537], to obtain that the coefficient of the $u_{\alpha} v_{\mu} w_{p}$-term of $q_{0}$, denoted by $q_{0}^{\alpha \mu p}$, is

$$
q_{0}^{\alpha \mu p}=\sum_{b \geq 1} T_{0 b}^{p} S_{\alpha \mu}^{b}=2 \sum_{b \geq 1} T_{0 b}^{p} F_{\alpha b}^{\mu},
$$

where $S_{\alpha \mu}^{b}=2 F_{\alpha a}^{\mu}$ is employed in [6, (6.4)]. On the other hand, by (1) and $p_{0}$ in (7), a similar calculation as in (9) yields

$$
\begin{aligned}
q_{0}^{\alpha \mu p} & =2\left\langle Q\left(Y_{\mu}, Z_{p}, X_{\alpha}\right), n_{0}\right\rangle \\
& =2\left(d S_{p \alpha}^{0}\left(Y_{\mu}\right)-\sum_{t} \theta_{p}^{t}\left(Y_{\mu}\right) S_{t \alpha}^{0}-\sum_{t} \theta_{\alpha}^{t}\left(Y_{\mu}\right) S_{p t}^{0}\right) \\
& =-2 \sum_{t} \theta_{p}^{t}\left(Y_{\mu}\right) S_{t \alpha}^{0}=-2 \theta_{p}^{\alpha}\left(Y_{\mu}\right) \\
& =4 F_{\alpha p}^{\mu},
\end{aligned}
$$

where the last equality follows by $[6,(4.18)]$. In conclusion, we derive

$$
F_{\alpha p}^{\mu}=\sum_{b} f_{p b} F_{\alpha b}^{\mu}
$$

where

$$
f_{p b}=T_{0 b}^{p} / 2,
$$

which is exactly $[6,(6.13)]$. Therefore, we may assume, as in $[6$, Proposition 11, p. 19], that

$$
F_{\alpha a+m_{1}}^{\mu}=F_{\alpha a}^{\mu}
$$

once we show that the matrix $\left(f_{p b}\right)$ is orthogonal. This is indeed true; the key is another of the 10 identities of Ozeki and Takeuchi [19, Part I, p. 530] defining an isoparametric hypersurface,

$$
\begin{aligned}
16 \sum_{a=0}^{m_{1}}\left(q_{a}\right)^{2}= & 16 G\left(\sum_{\alpha}\left(u_{\alpha}\right)^{2}+\sum_{\mu}\left(v_{\mu}\right)^{2}+\sum_{p}\left(w_{p}\right)^{2}\right) \\
& -\langle\nabla G, \nabla G\rangle,
\end{aligned}
$$


where $G=\sum_{a=0}^{m_{1}}\left(p_{a}\right)^{2}$. We can employ the commutative algebra scheme of Proposition 1 again on (14) to rewrite it as a polynomial homogeneous in all $p_{a} p_{b}$ with (homogeneous) polynomial coefficients of degree 2 , so that these coefficients are linear combinations of all $p_{a}$. Specifically, the coefficient of $\left(p_{0}\right)^{2}$ is

$$
16 \sum_{a=1}^{m_{1}}\left(r_{0 a}\right)^{2}-16\left(\sum_{\alpha}\left(u_{\alpha}\right)^{2}+\sum_{\mu}\left(v_{\mu}\right)^{2}+\sum_{p}\left(w_{p}\right)^{2}\right)+4\left\langle\nabla p_{0}, \nabla p_{0}\right\rangle,
$$

which is a linear combination of $p_{0}, p_{1}, \ldots, p_{m_{1}}$. Knowing that $r_{0 a}$ are functions of $w_{p}$ alone by (6) and (10), we invoke (7) and compare variable types to conclude that

$$
\sum_{a=1}^{m_{1}}\left(r_{0 a}\right)^{2}=\sum_{p=m_{1}+1}^{2 m_{1}}\left(w_{p}\right)^{2}
$$

where we agree that $p$ is indexed between $m_{1}+1$ and $2 m_{1}$. But then (6) for $r_{0 a}$ in terms of (15) says exactly that the matrix $\left(f_{p b}\right)$ is orthogonal.

Now with the index choice for $p$, we see that

$$
f_{a+m_{1} b}=\delta_{a b}
$$

by (11) and (13). That is, by (6), (10), and (12),

$$
r_{0 b}=2 \sum_{a} \delta_{b}^{a} w_{a+m_{1}}=2 w_{b+m_{1}}
$$

and, with the Einstein summation convention,

$$
\begin{aligned}
q_{0} & =r_{0 b} p_{b} \\
& =2\left(\delta_{b}^{a} w_{a+m_{1}}\right)\left(S_{\alpha \mu}^{b} u_{\alpha} v_{\mu}+S_{\alpha c+m_{1}}^{b} u_{\alpha} w_{c+m_{1}}+S_{\mu c+m_{1}}^{b} v_{\mu} w_{c+m_{1}}\right) .
\end{aligned}
$$

Hence, we have

$$
\sum_{a b c \alpha}\left(\delta_{b}^{a} w_{a+m_{1}}\right)\left(S_{\alpha c+m_{1}}^{b} u_{\alpha} w_{c+m_{1}}\right)=0
$$

or, equivalently, noting that $S_{\alpha c+m_{1}}^{a}=-F_{c+m_{1} a}^{\alpha}$ by [6, p. 18],

$$
\sum_{a c} F_{c+m_{1} a}^{\alpha} w_{c+m_{1}} w_{a+m_{1}}=0 .
$$


In other words, we have

$$
F_{c+m_{1} a}^{\alpha}=-F_{a+m_{1} c}^{\alpha} .
$$

Likewise, we have

$$
F_{c+m_{1} a}^{\mu}=-F_{a+m_{1} c}^{\mu} .
$$

LEMMA 1. If $m_{1}<m_{2}$, then the vectors

$$
\left(F_{\alpha 1}^{\mu}, F_{\alpha 2}^{\mu}, \ldots, F_{\alpha m_{1}}^{\mu}\right)
$$

for $1 \leq \alpha, \mu \leq m_{2}$, span $\mathbb{R}^{m_{1}}$.

Proof. This is [6, Proposition 7, p. 18].

LEMma 2. Let $m_{1}<m_{2}$. If (13), (16), and (17) hold, then the hypersurface is of OT-FKM type.

Proof. It suffices to show that $[6,(8.4)$, p. 28] holds. Then [6, Theorem 24, p. 36] (or see [7] for a conceptual proof of it) will establish the conclusion.

Since all the formulas are given in the proof of [6, Proposition 19], we will be brief and follow all notation there.

Employing equations [6, (8.5)-(8.9)], one establishes, by (13)-(17) with the Einstein summation convention, that

$F_{\alpha b}^{\mu}\left(-\theta_{a}^{b}+\theta_{a+m_{1}}^{b+m_{1}}\right)=\left(F_{\alpha a c}^{\mu}-F_{\alpha a+m_{1} c}^{\mu}\right) \theta^{c}+\left(F_{\alpha a c+m_{1}}^{\mu}-F_{\alpha a+m_{1} c+m_{1}}^{\mu}\right) \theta^{c+m_{1}}$.

Then the equations of $[6,(8.21)-(8.24)]$ imply that

$$
F_{\alpha a c}^{\mu}-F_{\alpha a+m_{1} c}^{\mu}=F_{\alpha a c+m_{1}}^{\mu}-F_{\alpha a+m_{1} c+m_{1}}^{\mu},
$$

so that $\theta_{a}^{b}-\theta_{a+m_{1}}^{b+m_{1}}$ is spanned only by $\theta^{c}+\theta^{c+m_{1}}, 1 \leq c \leq m_{1}$, by Lemma 1 above. This proves $[6,(8.4)]$.

We summarize what we have done so far in the following satisfying proposition.

Proposition 4. Let $m_{1}<m_{2}$. If $p_{0}, p_{1}, \ldots, p_{m_{1}}$ form a regular sequence, then the isoparametric hypersurface is of OT-FKM type. 


\section{§4. The dimension estimate when $m_{2} \geq 2 m_{1}-1$}

We now show that if $m_{2} \geq 2 m_{1}-1$, then the assumption in Proposition 4 holds so that the isoparametric hypersurface is of OT-FKM type. Henceforth, all homogeneous polynomials are regarded as being over the complex numbers.

We agree that $\mathbb{C}^{2 m_{2}+m_{1}}$ consists of points $(u, v, w)$ with coordinates $u_{\alpha}, v_{\mu}$, and $w_{p}$, where $1 \leq \alpha, \mu \leq m_{2}$ and $1 \leq p \leq m_{1}$. For $0 \leq k \leq m_{1}$, let

$$
W_{k}:=\left\{(u, v, w) \in \mathbb{C}^{2 m_{2}+m_{1}}: p_{0}(u, v, w)=\cdots=p_{k}(u, v, w)=0\right\} .
$$

We want to estimate the dimension of the subvariety $U_{k}$ of $\mathbb{C}^{2 m_{2}+m_{1}}$, where

$$
U_{k}:=\left\{(u, v, w) \in \mathbb{C}^{2 m_{2}+m_{1}}: \text { rank of the Jacobian of } p_{0}, \ldots, p_{k}<k+1\right\} .
$$

Similar to [6, p. 68], $p_{0}, \ldots, p_{k}$ give rise to a linear system of cones $\mathcal{C}_{\lambda}$ defined by

$$
c_{0} p_{0}+\cdots+c_{k} p_{k}=0
$$

with $\lambda=\left[c_{0}: \cdots: c_{k}\right] \in \mathbb{C} P^{k}$. The singular subvariety of $\mathcal{C}_{\lambda}$ is

$$
\mathscr{S}_{\lambda}:=\left\{(u, v, w) \in \mathbb{C}^{2 m_{2}+m_{1}}:\left(c_{0} S_{n_{0}}+\cdots+c_{k} S_{n_{k}}\right) \cdot(u, v, w)^{t r}=0\right\},
$$

where $\left\langle S_{n_{i}}(X), Y\right\rangle=\left\langle S(X, Y), n_{i}\right\rangle$ is the shape operator of the focal manifold $M_{+}$in the normal direction $n_{i}$; we have

$$
U_{k}=\bigcup_{\lambda} \mathscr{S}_{\lambda} .
$$

By Corollary 1, we wish to establish

$$
\operatorname{dim}\left(W_{k} \cap U_{k}\right) \leq \operatorname{dim}\left(W_{k}\right)-2
$$

for $k \leq m_{1}-1$ to verify that $p_{0}, p_{1}, \ldots, p_{m_{1}}$ form a regular sequence.

We first estimate the dimension of $\mathscr{S}_{\lambda}$. We break it into two cases. If $c_{0}, \ldots, c_{k}$ are either all real or all purely imaginary, then

$$
\operatorname{dim}\left(\mathscr{S}_{\lambda}\right)=m_{1}
$$

since $c_{0} S_{n_{0}}+\cdots+c_{k} S_{n_{k}}=c S_{n}$ for some unit normal vector $n$ and some nonzero real or purely imaginary constant $c$, and we know that the null space of $S_{n}$ is of dimension $m_{1}$. On the other hand, if $c_{0}, \ldots, c_{k}$ are not 
all real and not all purely imaginary, then similar to [6, p. 69], after a normal basis change, we can assume that $\mathscr{S}_{\lambda}$ consists of elements $(u, v, w)$ of the form $\left(S_{n_{1}^{*}}-\tau S_{n_{0}^{*}}\right) \cdot(u, v, w)^{t r}=0$ for some nonzero complex number $\tau$, relative to a new orthonormal normal basis $n_{0}^{*}, n_{1}^{*}, \ldots, n_{k}^{*}$ in the linear span of $n_{0}, n_{1}, \ldots, n_{k}$. That is, in matrix form,

$$
\left(\begin{array}{ccc}
0 & A & B \\
A^{t r} & 0 & C \\
B^{t r} & C^{t r} & 0
\end{array}\right)\left(\begin{array}{l}
x \\
y \\
z
\end{array}\right)=\tau\left(\begin{array}{ccc}
I & 0 & 0 \\
0 & -I & 0 \\
0 & 0 & 0
\end{array}\right)\left(\begin{array}{l}
x \\
y \\
z
\end{array}\right)
$$

where $x, y$, and $z$ are (complex) eigenvectors of (real) $S_{n_{0}^{*}}$ with eigenvalues 1, -1 , and 0, respectively; [6, Lemma 49, p. 64] ensures that we can assume that

$$
B=C=\left(\begin{array}{ll}
0 & 0 \\
0 & \sigma
\end{array}\right),
$$

where $\sigma$ is a nonsingular diagonal matrix of size $r$ by $r$, with $r$ the rank of $B$, and that $A$ is of the form

$$
A=\left(\begin{array}{ll}
I & 0 \\
0 & \Delta
\end{array}\right)
$$

where $\Delta=\operatorname{diag}\left(\Delta_{1}, \Delta_{2}, \Delta_{3}, \ldots\right)$ is of size $r$ by $r$, in which $\Delta_{1}=0$ and $\Delta_{i}, i \geq$ 2 are nonzero skew-symmetric matrices expressed in the block form $\Delta_{i}=$ $\operatorname{diag}\left(\Theta_{i}, \Theta_{i}, \Theta_{i}, \ldots\right)$ with $\Theta_{i}$ a 2 -by-2 matrix of the form

$$
\left(\begin{array}{cc}
0 & f_{i} \\
-f_{i} & 0
\end{array}\right)
$$

for some $0<f_{i}<1$. We decompose $x, y, z$ into $x=\left(x_{1}, x_{2}\right), y=\left(y_{1}, y_{2}\right), z=$ $\left(z_{1}, z_{2}\right)$ with $x_{2}, y_{2}, z_{2} \in \mathbb{C}^{r}$ (by abuse of notation, we do not distinguish column vectors from row vectors). Then (20) comes down to

$$
\begin{gathered}
x_{1}=-\tau y_{1}, \quad y_{1}=\tau x_{1}, \\
-\Delta x_{2}+\sigma z_{2}=-\tau y_{2}, \quad \Delta y_{2}+\sigma z_{2}=\tau x_{2}, \\
\Delta\left(x_{2}+y_{2}\right)=0 .
\end{gathered}
$$

It follows from the first set of equations of (22) that either $x_{1}=y_{1}=0$ or both are nonzero with $\tau= \pm \sqrt{-1}$. In both cases, by the second set of equations of (22), we have

$$
\left(\Delta^{2}-\tau^{2} I\right) x_{2}=(\Delta-\tau I) \sigma z_{2}, \quad\left(\Delta^{2}-\tau^{2} I\right) y_{2}=-(\Delta-\tau I) \sigma z_{2},
$$


which together with the third equation of (22) imply that $x_{2}=-y_{2}$, and so $z_{2}$ can be solved in terms of $x_{2}$ by the second set of equations of (22). (Note that, conversely, $x_{2}=-y_{2}$ can be solved in terms of $z_{2}$ when $\tau \neq \pm f_{i} \sqrt{-1}$ for all $i$, so that $z$ can be chosen to be a free variable in this case.) So, either $x_{1}=y_{1}=0$, in which case

$$
\operatorname{dim}\left(\mathscr{S}_{\lambda}\right)=m_{1}
$$

or both $x_{1}$ and $y_{1}$ are nonzero, in which case $y_{1}= \pm \sqrt{-1} x_{1}$ and so

$$
\operatorname{dim}\left(\mathscr{S}_{\lambda}\right)=m_{1}+m_{2}-r .
$$

Since eventually we must estimate the dimension of $W_{k} \cap U_{k}$, let us cut $\mathscr{S}_{\lambda}$ by

$$
0=p_{0}^{*}=\sum_{\alpha}\left(x_{\alpha}\right)^{2}-\sum_{\mu}\left(y_{\mu}\right)^{2} .
$$

Case 1: $x_{1}$ and $y_{1}$ are both nonzero. This is the case of nongeneric $\lambda \in \mathbb{C} P^{k}$. We substitute $y_{1}= \pm \sqrt{-1} x_{1}$ and $x_{2}$ and $y_{2}$ in terms of $z_{2}$ into $p_{0}^{*}=0$ to deduce that

$$
0=p_{0}^{*}=\left(x_{1}\right)^{2}+\cdots+\left(x_{m_{2}-r}\right)^{2}+z \text { terms; }
$$

hence, $p_{0}^{*}=0$ cuts $\mathscr{S}_{\lambda}$ to reduce the dimension by 1 . That is, by $(23)$,

$$
\operatorname{dim}\left(W_{k} \cap \mathscr{S}_{\lambda}\right) \leq\left(m_{1}+m_{2}-r\right)-1 \leq m_{1}+m_{2}-1,
$$

noting that $W_{k}$ is also cut out by $p_{0}^{*}, p_{1}^{*}, \ldots, p_{k}^{*}$. Meanwhile, similar to $[6$, p. 71], only a subvariety of $\lambda$ of dimension $k-1$ in $\mathbb{C} P^{k}$ assumes that $\tau= \pm \sqrt{-1}$. Therefore, by (24), an irreducible component $\mathcal{W}$ of $W_{k} \cap \bigcup_{\lambda} \mathscr{S}_{\lambda}$ over nongeneric $\lambda$ will satisfy

$$
\operatorname{dim}(\mathcal{W}) \leq \operatorname{dim}\left(W_{k} \cap \mathscr{S}_{\lambda}\right)+k-1 \leq m_{1}+m_{2}+k-2 .
$$

Case 2: $x_{1}=y_{1}=0$. This is the case of generic $\lambda$, where $\operatorname{dim}\left(\mathscr{S}_{\lambda}\right)=m_{1}$, so that an irreducible component $\mathcal{V}$ of $W_{k} \cap \bigcup_{\lambda} \mathscr{S}_{\lambda}$ over generic $\lambda$ will satisfy

$$
\operatorname{dim}(\mathcal{V}) \leq m_{1}+k \leq m_{1}+m_{2}+k-2
$$

as we may assume that $m_{2} \geq 2$. (The case $m_{1}=m_{2}=1$ is straightforward; see $[6$, p. 61].)

Putting these two cases together, we conclude that

$$
\operatorname{dim}\left(W_{k} \cap U_{k}\right) \leq m_{1}+m_{2}+k-2 .
$$


On the other hand, since $W_{k}$ is cut out by $k+1$ equations, we have

$$
\operatorname{dim}\left(W_{k}\right) \geq m_{1}+2 m_{2}-k-1
$$

Therefore,

$$
\operatorname{dim}\left(W_{k} \cap U_{k}\right) \leq \operatorname{dim}\left(W_{k}\right)-2
$$

when $k \leq m_{1}-1$, taking $m_{2} \geq 2 m_{1}-1$ into account.

In summary, we have established (19) for $k \leq m_{1}-1$, so that the ideal $\left(p_{0}, p_{1}, \ldots, p_{k}\right)$ is prime when $k \leq m_{1}-1$. Corollary 1 then implies that $p_{0}, p_{1}, \ldots, p_{m_{1}}$ form a regular sequence. It follows by Proposition 4 that the isoparametric hypersurface is of OT-FKM type.

$\S 5$. The case $\left(m_{1}, m_{2}\right)=(3,4)$

Recall that a point of $M_{+}$is of Condition A if the shape operators $S_{n}$ share the same kernel for all unit normal $n$. Conditions A and B were introduced and explored by Ozeki and Takeuchi [19, Part I, p. 541] in their construction of inhomogeneous isoparametric hypersurfaces in spheres. It was then established by Dorfmeister and Neher [9] (see also [8]) that Condition A alone implies that the isoparametric hypersurface is of OT-FKM type.

Theorem 1. Let $\left(m_{1}, m_{2}\right)=(3,4)$. Then there exist points of Condition $A$ on $M_{+}$; the isoparametric hypersurface is then of OT-FKM type.

Proof. We follow the notation in Section 4. Suppose that $M_{+}$is free of points of Condition A everywhere. Then one of the three pairs of matrices $\left(B_{1}, C_{1}\right),\left(B_{2}, C_{2}\right)$, and $\left(B_{3}, C_{3}\right)$ of the shape operators $S_{n_{1}}, S_{n_{2}}$, and $S_{n_{3}}$, similar to the one given in $(20)$, must be nonzero. By replacing $\left(B_{2}, C_{2}\right)$ by $\left(B_{3}, C_{3}\right)$, we may assume that one of $\left(B_{1}, C_{1}\right)$ and $\left(B_{2}, C_{2}\right)$ is nonzero in the neighborhood of a given point. For $k=m_{1}-1=2$, observe that in (24) if $r>0$ holds, then in fact

$$
\operatorname{dim}\left(W_{2} \cap \mathscr{S}_{\lambda}\right) \leq m_{1}+m_{2}-2
$$

If $r=0$, then $B_{1}^{*}=C_{1}^{*}=0$ and $A^{*}=I$ in $(20)$ for $S_{n_{1}^{*}}$. It follows that $p_{0}^{*}=0$ and $p_{1}^{*}=0$ cut $\mathscr{S}_{\lambda}$ in the variety

$$
\left\{(x, \pm \sqrt{-1} x, z): \sum_{\alpha}\left(x_{\alpha}\right)^{2}=0\right\} .
$$


$\left(B_{2}^{*}, C_{2}^{*}\right)$ of $S_{n_{2}^{*}}$ must be nonzero now. Since $z$ is a free variable in $(28)$, $p_{2}^{*}=0$ will have nontrivial $z$-terms

$$
\begin{aligned}
0=p_{2}^{*} & =\sum_{\alpha p} S_{\alpha p} x_{\alpha} z_{p}+\sum_{\mu p} T_{\mu p} y_{\mu} z_{p}+x_{\alpha} y_{\mu} \text { terms } \\
& =\sum_{\alpha p}\left(S_{\alpha p} \pm \sqrt{-1} T_{\alpha p}\right) x_{\alpha} z_{p}+x_{\alpha} x_{\mu} \text { terms }
\end{aligned}
$$

taking $y= \pm \sqrt{-1} x$ into account, where $S_{\alpha p}:=\left\langle S\left(X_{\alpha}^{*}, Z_{p}^{*}\right), n_{2}^{*}\right\rangle$ and $T_{\mu p}:=$ $\left\langle S\left(Y_{\mu}^{*}, Z_{p}^{*}\right), n_{2}^{*}\right\rangle$ are (real) entries of $B_{2}^{*}$ and $C_{2}^{*}$, respectively, and where $X_{\alpha}^{*}, 1 \leq \alpha \leq m_{2}, Y_{\mu}^{*}, 1 \leq \mu \leq m_{2}$, and $Z_{p}^{*}, 1 \leq p \leq m_{1}$ are orthonormal eigenvectors for the eigenspaces of $S_{n_{0}^{*}}$ with eigenvalues $1,-1$, and 0 , respectively; hence, the dimension of $\mathscr{S}_{\lambda}$ will be cut down by 2 by $p_{0}^{*}, p_{1}^{*}, p_{2}^{*}=0$, so that, again,

$$
\operatorname{dim}\left(W_{2} \cap \mathscr{S}_{\lambda}\right) \leq m_{1}+m_{2}-2
$$

noting that $p_{0}^{*}, p_{1}^{*}, p_{2}^{*}=0$ also cut out $W_{2}$.

In conclusion, for $k=2$,

$$
\operatorname{dim}\left(W_{k} \cap U_{k}\right) \leq \operatorname{dim}\left(W_{k} \cap \mathscr{S}_{\lambda}\right)+k-1 \leq m_{1}+m_{2}+k-3=6,
$$

while by (26),

$$
\operatorname{dim}\left(W_{k}\right) \geq 8
$$

so that

$$
\operatorname{dim}\left(W_{2} \cap U_{2}\right) \leq \operatorname{dim}\left(W_{2}\right)-2 .
$$

(Again, it suffices to consider the nongeneric case for the dimension estimate since the generic case contributes only dimension at most $m_{1}+k=5$, similar to what is detailed in Section 4.)

Meanwhile, (25) and (26) for $k \leq 1$ imply that

$$
\operatorname{dim}\left(W_{k} \cap U_{k}\right) \leq \operatorname{dim}\left(W_{k}\right)-2
$$

Therefore, it follows again by $(19)$ that $\left(p_{0}\right),\left(p_{0}, p_{1}\right)$, and $\left(p_{0}, p_{1}, p_{2}\right)$ are all prime ideals, and Corollary 1 asserts that $p_{0}, p_{1}, p_{2}, p_{3}$ form a regular sequence, so that Proposition 4 establishes that the isoparametric hypersurface is of the type constructed by Ozeki and Takeuchi [19, Part I], which has points of Condition A on $M_{+}$. This contradiction to the assumption made at the outset shows that, indeed, $M_{+}$has points of Condition A. 


\section{$\S 6$. Concluding remarks}

Knowing that $M_{+}$has points of Condition A for an isoparametric hypersurface with multiplicities $(7,8)$ of OT-FKM type (see [12]), one is tempted to apply the upper bound estimate $m_{1}+m_{2}+k-3$ in (30) and the lower bound estimate $m_{1}+2 m_{2}-k-1$ in (26) to the case, with $k=6$, to assert (19). This encounters apparent difficulties.

In fact, the upper bound cannot be improved to $m_{1}+m_{2}+k-4$ without understanding further properties pertaining to an isoparametric hypersurface with one of the three remaining multiplicity pairs, since otherwise $\left(m_{1}, m_{2}\right)=(4,5)$ for $k \leq 3$ would satisfy

$$
\operatorname{dim}\left(W_{k} \cap U_{k}\right) \leq \operatorname{dim}\left(W_{k}\right)-2,
$$

so that the same arguments as above would imply that the isoparametric hypersurface would be of OT-FKM type, which is not the case.

A detailed understanding of the number $r$ in (24) for the multiplicity pair $(7,8)$ would seem important.

\section{REFERENCES}

[1] U. Abresch, Isoparametric hypersurfaces with four or six distinct principal curvatures, Math. Ann. 264 (1983), 283-302.

[2] E. Cartan, Familles de surfaces isoparamétriques dans les espaces à courbure constante, Ann. Mat. Pura Appl. (4) 17 (1938), 177-191.

[3] - Sur des familles remarquables d'hypersurfaces isoparamétriques dans les espaces sphériques, Math. Z. 45 (1939), 335-367.

[4] - Sur quelque familles remarquables d'hypersurfaces, C. R. Congr. Math. Liège 1939, 30-41.

[5] — Sur des familles d'hypersurfaces isoparamétriques des espaces sphériques à 5 et à 9 dimensions, Rev. Univ. Tucuman Ser. A 1 (1940), 5-22.

[6] T. E. Cecil, Q.-S. Chi, and G. R. Jensen, Isoparametric hypersurfaces with four principal curvatures, Ann. of Math. (2) 166 (2007), 1-76.

[7] Q.-S. Chi, Isoparametric hypersurfaces with four principal curvatures revisited, Nagoya Math. J. 193 (2009), 129-154.

[8] — A new look at condition A, preprint, to appear in Osaka J. Math, arXiv:0907.0377v1[math.DG]

[9] J. Dorfmeister and E. Neher, Isoparametric triple systems of algebra type, Osaka J. Math. 20 (1983), 145-175.

[10] - Isoparametric hypersurfaces, case $g=6, m=1$, Comm. Algebra 13 (1985), 2299-2368.

[11] E. V. Ferapontov, Isoparametric hypersurfaces in spheres, integrable nondiagonalizable systems of hydrodynamic type, and $N$-wave systems, Differential Geom. Appl. 35 (1995), 335-369. 
[12] D. Ferus, H. Karcher, and H.-F. Münzner, Cliffordalgebren und neue isoparametrische Hyperflächen, Math. Z. 177 (1981), 479-502.

[13] E. Kunz, Introduction to Commutative Algebra and Algebraic Geometry, Birkhäuser, Boston, 1985.

[14] E. Laura, Sopra la propagazione di onde in un mezzo indefinito, Scritti matematici offerti ad Enrico D'Ovidio 1918, 253-278.

[15] T. Levi-Civita, Famiglie di superficie isoparametrische nell'ordinario spacio euclideo, Atti Accad. Naz. Lincei Cl. Sci. Fis. Mat. Natur. Rend. Lincei (9) Mat. Appl. 26 (1937), 355-362.

[16] R. Miyaoka, The Dorfmeister-Neher theorem on isoparametric hypersurfaces, Osaka J. Math. 46 (2009), 695-715.

[17] - Isoparametric hypersurfaces with $(g, m)=(6,2)$, preprint, 2009.

[18] H.-F. Münzner, Isoparametrische Hyperflächen in Sphären, I, Math. Ann. 251 (1980), 57-71; II, 256 (1981), 215-232.

[19] H. Ozeki and M. Takeuchi, On some types of isoparametric hypersurfaces in spheres, I, Tohoku Math. J. (2) 27 (1975), 515-559; II, 28 (1976), 7-55.

[20] B. Segre, Una Proprietá caratteristixca di tre sistemi $\infty^{1}$ di superficie, Atti Accad. Sci. Torino Cl. Sci. Fis. Mat. Natur. 29 (1924), 666-671.

[21] - Famiglie di ipersuperficie isoparametrische negli spazi euclidei ad un qualunque numero di demesioni, Atti Accad. Naz. Lincei Cl. Sci. Fis. Mat. Natur. Rend. Lincei (9) Mat. Appl. 27 (1938), 203-207.

[22] C. Somigliana, Sulle relazione fra il principio di Huygens e l'ottica geometrica, Atti. Accad. Sci. Torino Cl. Sci. Fis. Mat. Natur. 24 (1918-1919), 974-979.

[23] S. Stolz, Multiplicities of Dupin hypersurfaces, Invent. Math. 138 (1999), 253-279.

\author{
Department of Mathematics \\ Washington University \\ St. Louis, Missouri \\ USA \\ chi@math. wustl.edu
}

